\title{
Uncertainty in coarse conservation assessments hinders the efficient achievement of conservation goals.
}

\author{
Virgilio Hermoso and Mark J. Kennard
}

Australian Rivers Institute and Tropical Rivers and Coastal Knowledge, National Environmental Research Program Northern Australian Hub, Griffith University, Nathan, Queensland, 4111, Australia

Corresponding author: Virgilio Hermoso

Email: virgilio.hermoso@gmail.com

Ph: (061) 0737355291

Fax: (061) 0737357615

Current address: Australian Rivers Institute and Tropical Rivers and Coastal Knowledge, National Environmental Research Program Northern Australian Hub, Griffith University, Nathan, Queensland, 4111, Australia. 


\begin{abstract}
Conservation planning is sensitive to a number of scale-related issues, such as the spatial extent of the planning area, or the size of units of planning. An extensive literature has reported a decline in efficiency of conservation outputs when planning at small spatial scales or when using large planning units. However, other key issues, such as the grain size used to represent the spatial distribution of conservation features. Here, we evaluate the effect of grain size of species distribution data vs. size of planning units on a set of performance measures describing efficiency (ratio of area where species are represented/ total area needed), rate of commission errors (species erroneously expected to occur), representativeness (proportion of species achieving the target) and a novel measure of overall conservation uncertainty (integrating commission errors and uncertainty in the actual locations where species occur). We compared priority areas for the conservation of freshwater fish in the Daly River basin (northern Australia). Our study demonstrates that the effect of grain size of species distribution data was more important than planning unit size on conservation planning performanceeonservation planning carried out using low resolution species distribution maps makes the achievement of conservation goals very difficult irrespective of the size of planning units used in the assessment, with an increase in commission errors up to $80 \%$ and conservation uncertainty over $90 \%$ when coarse data were used. This was more pronounced for rare than common species, where the mismatch between coarse representations of biodiversity patterns and the smaller areas of actual occupancy of species was more evident. Then, sSpecial attention should be paid to the high risk of misallocation of limited budgets when planning in heterogeneous or disturbed environments, where biodiversity is patchily distributed, or when planning with for conservation of rare species.
\end{abstract}

Keywords: Commission error, grain size, MARXAN, priority area, uncertainty, freshwater fish. 


\section{Introduction}

Biodiversity conservation usually competes with other human uses and interests so the identification of priority areas where conservation management actions can be effectively carried out (conservation planning) is a crucial task. In this sense, conservation planning is a spatially explicit task that involves many spatial-related decisions, such as establishing the extent of the planning area (e.g., continent, or river catchment), the size and shape of the units of planning or the grain size of the biodiversity data used in the planning process (Mills et al. 2010). However, these decisions concerning spatial scale can have important implications for the outputs of conservation planning assessments and hence the efficient use of limited resources for conservation management.

The spatial extent of conservation planning assessments has varied from global (Brooks et al. 2004), continental (Carwardine et al. 2008) or finer regional scales such as individual river basins (Hermoso et al. 2011a) or islands (Payet et al. 2010). The spatial extent of the planning exercise influences not only the spatial allocation of priority areas, but also the efficiency of the conservation plan and feasibility of its implementation (Wiersma 2007; Vazquez et al. 2008). Broad-scale assessments outperform small-scale ones in terms of efficiency. For example, Kark et al. (2009) found that the same conservation targets could be achieved at half the cost if a fully coordinated (multicountry) conservation plan was carried out across the Mediterranean basin. However, such broadscale plans often require coordinated efforts among different regional governments and countries which can hinder its application. Planning unit shape varies according to the realm in which the conservation planning process is carried out (e.g. regular grid cells or hexagons in terrestrial and marine planning, versus irregular polygons such as sub-catchments in freshwater planning). In contrast, the choice of planning unit size is often arbitrary (Warman et al. 2004), although planning units tend to be larger in assessments of extensive regions and smaller in more localised studies (Pressey and Logan 1998). Determining the appropriate shape and size of planning units is an important consideration in conservation planning because it also influences the spatial distribution of priority areas and their efficiency. Previous studies have reported a decrease in efficiency (defined as the ratio of the total area occupied by each species to the total conservation priority area) when using large planning units. This is mainly due to the lack of fit between the species area of occupancy and the boundaries of planning units, which can result in the selection of areas where the species of interest do not occur (Pressey and Logan 1995; 1998; Pressey et al. 1999; Rouget 2003). The use of different planning unit sizes has also proven to affect the identification of conservation priority areas (i.e. priority maps change with different planning unit sizes) (Rouget 2003; Warman et al 2004).

Despite the comprehensive scientific literature devoted to evaluating the effect of the spatial extent of the planning area and planning unit size and shape on conservation outputs, other important spatial issues have not been adequately assessed. For example, little is known about the effect of the 
grain size used to represent the spatial distribution of biodiversity surrogates (e.g. species or environmental classes). This is a different issue to planning unit size, since different grain sizes of species distribution data can be used on the same set of planning units (for example, see Figure 1a). Although fine-grained data on biodiversity surrogates are desirable due to their greater information content and hence precision of outcomes (Pressey and Bedward 1991; Rouget 2003; Banks and Skilleter 2007), conservation assessments are usually undertaken at coarse resolution due to limited data quality and quantity. So the use of coarse grain data is normally a consequence of data availability rather than a conservation planner or stakeholders' decision. Given that data gathering is an expensive and time-consuming task, conservation practitioners are usually forced to use the limited available data. These data sources often offer a biased picture of biodiversity patterns towards well studied areas (e.g., data records from birdwatchers) and are prone to commission or omission errors (Loiselle et al. 2003; Rondinini et al. 2006, Hermoso et al. 2011b). Commission or type I errors arise when species are erroneously expected to be present, which leads to false representation as the areas selected for conservation do not contain the expected species. Omission or type II errors occur when species are not detected, which may result in the selection of areas which are unnecessarily large or more expensive to manage than needed (Rondinini et al. 2006; Wilson et al. 2005). Coarse representations of species distributions tend to overestimate the species' area of occupancy, and are therefore prone to commission errors (Rondinini et al. 2006). This is especially the case for rare or patchily distributed species (e.g., in highly fragmented areas due to human perturbations). Coarse grain sizes also convey an increase in uncertainty in the spatial distribution of species. For example, a species could be expected to occur throughout a large patch if using a coarse grain size to represent its distribution, but only occupy a small portion of the patch. These issues have clear implications for the effective implementation of management actions to conserve biodiversity. Uncertainty concerning the precise distribution of species also makes it difficult to decide where to implement conservation actions or potentially very expensive if entire expected areas of occupancy (whole patch) had to be managed. Importantly, these two issues that arise from coarse conservation assessments and that could hinder the achievement of efficient conservation outcomes have not yet been explored.

Here we evaluate the effect of grain size on the efficiency, representativeness (adequate representation of all species), commission errors and conservation uncertainty (derived from species distribution uncertainty) of priority areas identified using a systematic conservation planning approach. We focus our study on freshwater fish biodiversity in the Daly River basin, northern Australia, an area with important ecological, cultural and economic values (Chan et al. 2010). We evaluate the role of two different sources of grain size variation: i) grain size of planning units used for conservation prioritisation and ii) grain size used to represent the spatial distribution of species. We use the outcomes of our study to provide critical guidance to conservation researchers and practitioners on the influence of grain size on conservation assessments. 


\section{Methods}

\subsection{Study area and species distribution data}

The Daly River basin (Fig. 1b) encompasses $53000 \mathrm{~km}^{2}$ and is in relatively good environmental condition compared to other major rivers in Australia. The dominant land-uses are low density cattle grazing and conservation areas (including a few major national parks), although small parts of the catchment have been cleared for more intensive land-uses such as urbanization, pasture and agriculture. The Daly River is currently unregulated, with only a small volume of groundwater extracted annually for agriculture, but there is considerable pressure for further agricultural development and water demand (Chan et al. 2010).

Fish species distribution data was sourced from Kennard (2010). This database contained continuous predictions of the spatial distribution for 104 species of freshwater fish across northern Australia derived from Multivariate Adaptive Regression Splines models (Leathwick et al. 2005) at a fine scale (average area of predictive polygons was $3.6 \mathrm{~km}^{2}$ ). The predictive model was built on a data set of 1609 presence-only records and validated using an independent data set of 719 presenceabsence records (see Hermoso et al. (2011c) for more details on predictive models). Predictions for each of the 46 freshwater fish species occurring in 14587 predictive units for the Daly River basin were extracted from this data base and used for subsequent analyses.

\subsection{Identification of priority areas}

Priority areas for conservation of freshwater fish in the Daly River basin were identified using the software MARXAN (Ball et al. 2009). MARXAN uses a simulated annealing optimization algorithm to find an optimal combination of areas to represent each species at a given target level of occurrence (e.g., $100 \mathrm{~km}^{2}$ of river catchment, hereafter termed conservation target). By using the principle of complementarity (gain in representation of biodiversity when a site is added to an existing set of planning units) MARXAN tries to find an optimum set of planning units that achieve the target representation for each species at the minimum cost. This is done by trying to minimize an objective function (Formula 1) that includes the cost of planning units in the solution and other penalties for not achieving the conservation target for all the species (Feature Penalty, weighted by Species' Penalty Factor, SPF). We used a high SPF across all analyses ( $\mathrm{SPF}=100)$ to ensure all the species achieved the target. Additional penalties and costs can be specified in the objective function to force the spatial aggregation of planning units included in the solution and ensure high internal connectivity within priority areas, and to favour the selection of priority areas that are potentially cheapest to manage. Given our special interest in exploring the effect of grain size and the lack of accurate estimates of 
conservation cost for the Daly River, we used a constant cost across the study area (all the planning units were assigned a cost of 1) and we did not include the connectivity term in the objective function. If heterogeneous cost data and connectivity had been used, the MARXAN selection processes would have been forced to avoid expensive areas and select connected planning units (see Hermoso et al. 2011a), and it would have been difficult to distinguish between the effect of local patterns in cost and connectivity from the effect of grain size. We made these simplifications to explore the effect of the different grain sizes although we acknowledge that better estimates of conservation or management cost and the incorporation of connectivity issues would be desirable for the actual design and implementation of a conservation plan for a particular area.

$$
\text { Objectivefunction }=\sum_{\text {planningunits }} \operatorname{Cos} t+S P F \sum_{\text {features }} \text { Feature Penalty (Formula 1) }
$$

The optimization algorithm in MARXAN was run 100 times (1M iterations each run) to find 100 near-optimal solutions, the best of which was used in the analyses below (called best solution hereafter).

\subsection{Influence of grain size on conservation outputs}

Five different scenarios were designed to evaluate the relative importance of variation in the grain size of planning units used for conservation prioritisation and the grain size used to represent the spatial distribution of species (Fig. 1a, Appendix A). These conservation scenarios were: i) constant fine planning unit size, with varying grain sizes of species distributions, ii) constant coarse planning unit size, with varying grain sizes of species distributions, iii) constant fine species distribution grain size, with varying grain sizes of planning units, iv) constant coarse species distribution grain size, with varying grain sizes of planning units, and v) a mixed scenario (varying both planning unit and species distribution grain sizes at the same time). Separate analyses were run for the two most common types of species distribution data used in conservation planning: species' occurrence and area of occupancy within each planning unit.

Planning units. Rather than using equal-sized grid cells as planning units as is usually done in terrestrial and marine conservation assessments, we used sub-catchments as they are more appropriate and ecologically relevant for freshwater ecosystems. We used ARC Hydro (Maidment 2002) for ArcGIS 9.3 (ESRI 2002) to delineate planning units from a 9 second digital elevation model. Planning units were separately delineated for eleven grain sizes (finest grain size and 10 coarser test grain sizes) (Table 1). Each planning unit included the portion of river length between two consecutive 
nodes or river connections and its contributing area (ranging from 4 to $1121 \mathrm{~km}^{2}$ on average for the finest and coarsest grain sizes, respectively). Planning units were spatially nested across different grain size scenarios, so a coarse planning unit contained a number of finer size planning units (Fig. 1).

Species distributions. We translated the predicted spatial distribution of each species into the finest grain set of planning units. A species was assumed to be present in a planning unit (and occupy its entire area) whenever it appeared in at least one of the predictive polygons described earlier. This finest grain size of species distribution data was used to scale up predictions to the ten coarser planning unit grain sizes. In this way, whenever a species was present in a finer scale planning unit contained within a coarser planning unit, that species was assumed to occupy the entire extent of the coarser planning unit. This approach had been used elsewhere to re-scale predictions in conservation planning studies (e.g., Conroy and Noon 1996; Justus et al. 2007). We then assumed each of them to be the best available representation of species distributions for each scenario (i.e. as if no better information was available).

The use of coarse grained data to represent species distributions necessarily results in higher uncertainty in their actual spatial distribution, especially for rare species or highly fragmented populations. For example, the spatial extent of patchily distributed species will be overestimated using coarse grain sizes and will be expected to occur in areas where they are not present or areas with low habitat suitability. At each grain size the uncertainty of species distributions (hereafter termed distribution uncertainty) was quantified for both species' occurrence and area of occupancy data as the proportion of expected occurrence or area of occupancy that was actually true at the finest grain size averaged across species [distribution uncertainty $=1-$ (occurrence or area occupancy at finest grain size / expected occurrence or area occupancy at coarser grain size); Fig. 2a].

Conservation targets. A constant target of species representation was used across all species (i.e. the same number of species' occurrences or area of occupancy). Given that the number of occurrences changed with grain size (all occurrences within a coarser planning unit became one) the target was adjusted accordingly to the new grain size to represent approximately $6 \%$ of total number of planning units (Table 1). This was not an issue for species distribution data expressed as area of occupancy, so a constant value was used in this case (Table 1).

Similar to Rouget (2003), we compared the results from the different conservation planning scenarios against the finest planning unit and species distribution grain size (representing the best potential result achievable or "true situation"). All best solutions from the conservation prioritization analyses run at different grain sizes were translated into the finest grain size of planning units, so they could be compared. For example, whenever a coarse planning unit was included in a best solution, all the finest ones contained within it were marked as included in the coarse best solution. 
We used four different performance measures to evaluate the effect of grain size of planning units and grain size of species distribution data on conservation outputs.

a) Efficiency. For species' presence-absence data we measured the efficiency of best solutions for each species as the ratio of the number of planning units where each species was present within the best solution to the total number of planning units in the best solution. For species' area of occupancy data we measured efficiency as the ratio of the total area occupied by each species to the total area of the best solution. The total area or number of planning units occupied within the best solution is referred as representation hereafter.

b) Commission error rate. Commission errors for the range of grain sizes of planning units and species distribution data were calculated by comparison to the true situation. This was estimated as the proportion of the expected species' representation at a given scale that would not be actually achieved due to overestimation of species occurrences [commission error $=1-(($ representation fine scale - representation coarse scale) / representation coarse scale); Fig. 2b].

c) Conservation uncertainty. Both commission errors and the increase in uncertainty of species distributions when planning at coarse grain sizes may have negative consequences for the practical conservation of species. We integrated loss in efficiency and distribution uncertainty into a single conservation uncertainty index [conservation uncertainty $=(1+$ commission error $) *$ distribution uncertainty] to evaluate the influence of different grain sizes on conservation outputs. This index ranges from $0-1,0$ indicating solutions with the lowest overall uncertainty (low commission errors and low distribution uncertainty) and 1 indicating high uncertainty in the spatial allocation of species within priority areas and high commission errors.

d) Proportion of species that achieved the conservation target. We measured the proportion of species that achieved the conservation target level for each best solution. As in previous performance measures, best solutions from different grain sizes were translated into the finest one and a species was considered to achieve the target either when representation in best solution > target level used or representation $=$ maximum area of occupancy predicted at finest grain size. Given that a common target level was used across all the species, this could exceed the actual area of occupancy for some rare species. Therefore, a species was also considered to achieve the target whenever its entire predicted area of occupancy was included in the best solution.

We used Generalized Linear Models (GLM) analyses to test for significant effects of grain size (of planning units or species distribution data, depending on the conservation scenario) on the variation of performance measures for each of the five conservation scenarios. We ran independent analyses for each performance measure (dependent variable), with grain size and species identity as explanatory factors. A gamma distribution of errors with an identity link function was used across all 
the GLM models. We also explored if the effect of grain size was similar across species (all species are equally affected), using a "homogeneity of slopes" analysis . The homogeneity of slopes is tested by introducing the interaction term (species $x$ grain size) in each GLM model. Lack of significance of this interaction term indicates no significant differences in the slope of the relationship between the dependent and the continuous predictor (grain size) across factor levels (species).

\section{Results}

The effect of increasing grain size on the uncertainty in species distributions (averaged across all species) increased in a curvilinear fashion up to a maximum of $80.2 \%$ and $83.1 \%$ for occurrence and abundance data, respectively, at the coarsest grain size (Appendix B). This increase was stronger for rare than common species (e.g., $96.1 \%, 79.7 \%$ and $2.1 \%$ for Neoarius graeffei, Toxotes chatareus and Leiopotherapon unicolor, respectively) due to the higher expected frequency of occurrence or area of occupancy of rare species with increasing grain size compared with common species (Fig. 3).

There was little difference between species' occurrence and area of occupancy data in the effect of grain size on all performance measures (Table 2). For this reason and the sake of simplicity we refer hereafter to results based on area of occupancy data only. Whenever fine grained planning units or species distribution data were used to identify conservation priority areas, efficiency in representing species was usually higher compared to coarse grained data, but it declined around $27 \%$ on average from the finest to the coarsest grain sizes of the other parameter (Table 2, Fig. 4a). In contrast, use of coarse grained data for one parameter resulted in insensitivity to variation in the grain size of the other parameter (for example efficiency for constant coarse planning units was 0.15 and did not vary appreciably with increasing species distribution grain sizes, Fig. 4a).

Commission errors increased markedly with increasing grain size for all scenarios (up to $93 \%$ for the "constant fine distribution" scenario), except for the "constant coarse distribution" scenario which always had high commission errors (Table 2, Fig. 4b). These results indicate that there was up to $93 \%$ of the expected species' representation that would not actually be achieved. These results also indicate that when using coarse grained data for one parameter (i.e. planning units or species distributions), the probability of commission errors increases, irrespective of the grain size of the other parameter. Overall conservation uncertainty (which combines both commission error and distribution uncertainty) followed a similar pattern to commission errors for all the scenarios, increasing by up to $97 \%$ for the "Constant fine distribution" scenario, if the coarsest planning unit grain size had been used instead of the finest one (Table 2, Fig. 4c).

Commission errors arising from the use of progressively coarser scale species distribution data resulted in progressively fewer species achieving the representation target when analysed at the finest 
scale for the "constant planning unit" scenarios (both fine and coarse) and the "mixed" scenario (Fig. 4d). The decline in the proportion of species that achieved the target was up to $70 \%$ (i.e. only $30 \%$ of species achieved the target when using the coarsest grain size). This indicates that independently of the planning unit grain size used, the proportion of species that achieve the target is strongly influenced by the coarseness of the species distribution data used. This result was confirmed by the "constant fine and coarse distribution" scenarios (Fig. 4d), where the proportion of species that achieved the target remained almost invariant across different planning unit grain sizes, but with very different results. The proportion of species that achieved the target was very high (close to 100\%) when fine distribution data was used, while it remained very low $(<25 \%)$ when coarse distribution data was used (Fig. 4d).

These results were supported by GLM analyses, where grain size had significant effects on efficiency for all scenarios except for the coarse scenarios ("constant coarse planning unit" and "constant coarse distribution"; Appendix C). Grain size had significant effects on commission errors for all scenarios except for the "constant coarse distribution" scenario and had significant effects on conservation uncertainty across all tested scenarios (Appendix C). The analysis of homogeneity of slopes showed the interaction term to be always significant, demonstrating species-specific responses to changes in grain size for all performance measures (Appendix C). These changes tended to be stronger for rare species than for common ones (Fig. 5).

\section{Discussion}

Conservation assessments are often carried out at broad spatial extents and rely on coarse resolution data due to constraints on data quality or budgetary and time limitations (Groves et al. 2002). Although some studies indicate that coarse grained biodiversity surrogates are suitable for conservation planning in homogeneous and relatively pristine areas (Rouget 2003), there is a general agreement that finer-scale data should ideally be used where available (Pressey and Logan 1995; 1998; Rodrigues and Gaston 2001; Banks and Skilleter 2007). Previous literature has focused on the evaluation of the effect of the size of planning units on the efficiency of priority areas to represent biodiversity (Pressey and Logan 1994; 1995; Rouget 2003). However, little is known about other spatial related issues, such as the effect of varying resolution species distribution data, or the implications of these factors on other measures of the performance of conservation outputs apart from the well-studied effects on efficiency (Wiersma 2007; Vazquez et al. 2008). Here, we show that the influence of the choice of grain size of both planning units and species distribution data extends beyond assessment of efficiency and that the achievement of conservation goals could be seriously compromised by commission errors and uncertainty in the distribution of species associated with coarse grained assessments. We demonstrate this by using species distribution data rather than coarse 
biodiversity surrogates based on land types, vegetation classes or other environmental classifications as previously done (Pressey and Logan 1994). To our knowledge, this is the first time that these issues have been addressed for freshwater ecosystems.

Previous studies have reported a decrease in efficiency with increasing planning unit size (e.g. Pressey and Logan 1994, 1998, Pressey et al. 1999). This decline has been primarily related to the lack of fit between the boundaries of planning units and the spatial extent of species' area of occupancy (Pressey and Logan 1995). Large planning units will usually contain areas where the species are not present, so their distributions patterns could be better represented using finer-scale planning units and hence lead to greater efficiency in selection of priority areas. Other factors such as the nestedness of species distributions have also been cited as an important factor explaining this reduction in efficiency when using comparatively large planning units (Pressey and Logan 1995). Nhancale \& Smith showed the effect of planning unit size to decrease when cost and connectivity constrains were applied. Here we argue that the resolution of species distributions should also be considered as an additional factor with strong implications for conservation assessments. Our study clearly demonstrated that the effect of grain size of species distribution data was more important than planning unit size on conservation planning performance. We found that different planning unit sizes had no significant effect on efficiency, and commission error rates when coarse species distribution data is used ("constant coarse distribution" scenario). Moreover, we found significant changes in commission errors when the size of planning units was maintained constant and the grain size of species distribution varied (up to 93\% commission errors when using the coarsest species distribution data). In addition, the proportion of species achieving the representation target was highly dependent on the grain size of the species distribution data but insensitive to planning unit size. The effect of cost can be ruled out from these results given that we found similar patterns when a different surrogate of cost was used (planning unit's area, data not shown).

We also calculated for the first time an overall performance measure of conservation uncertainty by integrating commission errors and uncertainty in the actual locations where species occur. This provides important information on the effects of grain size when planning for conservation of rare species with restricted distribution ranges (Andelman et al. 2002) or planning in disturbed landscapes where biodiversity is distributed in fragmented patches (Rouget 2003). In these cases where there is a mismatch between the-coarse representations of biodiversity patterns and the smaller areas of actual occupancy of species-, species' occurrences are overestimated leading to the inflated commission errors and low representativeness that we report here. Moreover, coarse resolution maps entail high uncertainty on species' spatial occurrences. This makes the planning and implementation of management actions for conservation, which are usually required when planning in perturbed landscapes, more difficult and potentially inefficient. The limited budgets available for 
conservation constrain the total area that can be effectively managed (e.g., implementation of rehabilitation programs). This entails a high risk of misallocating the management actions in areas where the targeted species do not actually occur; thereby missing the expected benefits from those management actions (Carwardine et al. 2008). We demonstrate that this happens even when using irregular planning units specifically derived to accommodate distribution of freshwater biodiversity (Linke et al. 2007). Regular-shaped planning units (squared or hexagonal grids) are normally used in other realms, like terrestrial or marine planning. This might worsen the results due to increased lack of fit between species actual occurrences and the surrogates used to represent them given to the less ecologically sound selection of planning units' boundaries.

Despite the benefits of using fine resolution data in conservation planning, it is not a common practice due to cost constraints. Data gathering to refine species' distributions can be expensive and time-consuming, and in many cases may not be possible (Regan et al. 2009). Acknowledging this limitation, some new advances in predictive modelling and research on different species mapping methods might help fulfil the necessity of fine resolution species distribution maps. Bombi et al. (2011) showed the pros and cons of different methods used to obtain distribution maps for conservation assessment. Stockwell and Peterson (2003) compared different methods of mapping biodiversity for gaining spatial resolution when limited data are available. They found species distribution predictive models produced more reliable and fine resolution data than the aggregation of species occurrences records or the use of vegetation surrogates. Araújo et al. (2005) and Barbosa et al. (2009) downscaled coarse distribution maps for different species in Europe by using predictive models fitted at coarse grain size to make predictions at finer resolutions, reporting good predictive performances.. However, these methods must be used cautiously given that additional research is needed to better understand the potential errors in downscaled predictions (Wiens and Bachelet 2010).

Uncertainty in conservation planning has been associated in part with inaccuracies in data used for the identification of priority areas (Regan et al. 2009). Key issues include uncertainties due to the grain size of planning units as well as the grain size of species distribution data used in conservation planning assessments. Our sensitivity analyses demonstrated the implications of choices concerning grain size on the efficiency, commission errors, overall conservation uncertainty and representativeness of priority areas for conservation. We therefore recommend that: 1) conservation planners should utilise the finest species distribution data available, particularly when planning for rare species or in fragmented landscapes where the limitations of coarse grain sizes are likely to be greatest; 2) whenever fine grained species distribution data are available, the use of coarse planning units should be avoided, 3) a sensitivity analyses such as demonstrated here should be undertaken to confirm that the effects of choices of grain size on conservation performance (as shown here for freshwater fish) are transferable to other taxonomic groups and/or realms. 


\section{Acknowledgements}

We thank J. Stein and D. Ward for their help with the environmental characterization of planning units, B. Pusey, D. Burrows, P. Bayliss and J. Boyden for assistance with compilation of fish and waterbird data and S. Linke for discussions in the initial planning stages of this paper. We acknowledge the Australian Government Department of Sustainability, Environment, Water, Population and Communities, the National Water Commission, the Tropical Rivers and Coastal Knowledge (TRaCK) Research Hub, and the Australian Rivers Institute, Griffith University, for funding this study. 


\section{References}

Andelman, S.J., Willig, M.R., 2002. Alternative configurations of reserves for Paraguayan bats: Considerations of spatial scales. Conservation Biology 16, 1352-1363.

Araújo, M.B., Thuiller, T., Williams P.H., Reginster, I., 2005. Downscaling European species atlas distributions to a finer resolution: implications for conservation planning. Global Ecology and Biogeography 14, 17-30.

Ball, I.R., Possingham, H.P., Watts, M., 2009. Marxan and relatives: Software for spatial conservation prioritisation. Pages 185-195 in Moilanen, A., K. A. Wilson, and H. P. Possingham, editors.Spatial conservation prioritisation: Quantitative methods and computational tools. Oxford University Press, Oxford, UK.

Banks, S. A., Skilleter, G.A., 2007. The importance of incorporating fine-scale habitat data into the design of an intertidal marine reserve system. Biological Conservation 138, 13-29.

Barbosa, A.M., Real, R., Vargas J.M., 2009. Use of Coarse-Resolution Models of Species' Distributions to Guide Local Conservation Inferences. Conservation Biology 24, 1378-1387.

Bombi, P., Luiselli L., D’Amen M., 2011. When the method for mapping species matters: defining priority areas for conservation of African freshwater turtles. Diversity and Distributions 17, 581-592.

Brooks, T.M., Bakarr, M.I., Boucher, T., Da Fonseca, G.A.B., Hilton-Taylor, C., Hoekstra, J.M., Moritz, T., Olivier, S., Parrish, J., Pressey, R.L., Rodrigues, A.S.L., Sechrest, W., Stattersfield, A., Strahm, W., Stuart, S.N., 2004. Coverage provided by the global protected-area system: Is it enough? Bioscience 54, 1081-1091.

Carwardine, J., Wilson, K.A., Watts, M., Etter, A., Klein, C. J., Possingham, H. P., 2008. Avoiding costly conservation mistakes: the importance of defining actions and cost in spatial prioritization setting. PLoS ONE 3, e2586. doi:10.1371/journal.pone.0002586.

Chan, T., Hart, B., Kennard, M.J., Pusey, B.J., Shenton, W., Douglas, M., Valentine, E., Patel, S., 2010. Bayesian network models for environmental flow decision-making in the Daly River, Northern Territory, Australia. River Research and Applications DOI: 10.1002/rra.1456.

Conroy, M.J., Noon B.R., 1996. Mapping of species richness for conservation of biological diversity: conceptual and methodological issues. Ecological Applications 6, 763-773.

ESRI, 2002. ArcGIS. Environmental Systems Research Institute, Redlands, CA. 
Groves, C.R., Jensen, D.B., Valutis, L.L., Redford, K.H., Shaffer, M.M., Scott, J.M., Baumgartner, J.V., Higgins, J.V., Beck, M. W., Anderson, M.G., 2002. Planning for biodiversity conservation: putting conservation science into practice. BioScience 52, 499-512.

Hermoso, V., Linke, S., Prenda, J., Possingham, H.P., 2011a. Addressing longitudinal connectivity in the systematic conservation planning of fresh waters. Freshwater Biology 56, 57-70.

Hermoso, V., Januchowski, S., Linke, S., Possingham, H.P., 2011b. Reference vs. present-day condition: early planning decisions influence the achievement of conservation objectives. Aquatic Conservation: Marine and Freshwater Research 21, 500-509.

Hermoso, V., Kennard, M.J., Linke, S., 2011c. Integrating multi-directional connectivity requirements in systematic conservation planning to prioritize fish and waterbird habitat in freshwater systems. Diversity and Distribution (in press).

Justus, J., Fuller, T., Sarkar, S., 2007. Influence of representation targets on the total area of conservation-area networks. Conservation Biology 22, 673-682.

Kark, S., Levin, N., Grantham, H.S., Possingham, H.P., 2009. Between-country collaboration and consideration of costs increase conservation planning efficiency in the Mediterranean Basin. Proceedings of the National Academy of Sciences 106, 15368-15373.

Kennard, M.J., 2010. Identifying high conservation value aquatic ecosystems in northern Australia. Interim Report for the Department of Environment, Water, Heritage and the Arts and the National Water Commission. Tropical Rivers and Coastal Knowledge (TRaCK) Commonwealth Environmental Research Facility, Charles Darwin University, Darwin. ISBN: 978-1-921576-23-2.

Leathwick, J.R., Rowe, D., Richardson, J., Elith, J., Hastie, T., 2005. Using multivariate adaptive regression splines to predict the distribution of New Zealand's freshwater diadromous fish. Freshwater Biology 50, 2034-2052.

Loiselle, B.A., Howell, C.A., Gaham, C.H., Goerck, J.M., Brooks, T., Smith, K.G., Williams, P.H., 2003. Avoiding pitfalls of using species distribution models in conservation planning. Conservation Biology 17, 1591-1600.

Maidment, D.R., 2002. Arc Hydro: GIS for Water Resources. ESRI Press, Redlands, CA. Mills, M., Pressey, R.L., Weeks, R., Foale, S., Ban, N.C., 2010. A mismatch of scales: challenges in planning for implementation of marine protected areas in the Coral Triangle. Conservation Letters 3, 291-303. 
Moilanen, A., Wintle, B.A., Elith, J., Burgman, M., 2006. Uncertainty analysis for regional-scale reserve selection. Conservation Biology 20, 1688-1697.

Payet, K., Rouget, M., Lagabrielle, E., Esler, K. J., 2010. Measuring the effectiveness of regional conservation assessments at representing biodiversity surrogates at a local scale: A case study in Réunion Island (Indian Ocean). Austral Ecology 35, 121-133.

Pressey, R.L., Logan, V.S., 1994. Levels of geographical subdivision and its effects on assessments of reserve coverage: a review of regional studies. Conservation Biology 8, 1037-1046.

Pressey, R.L., Logan, V.S., 1995. Reserve Coverage and Requirements in Relation to Partitioning and Generalization of Land Classes: Analyses for Western New South Wales. Conservation Biology 9, 1506-1517.

Pressey, R.L., Logan, V.S., 1998. Size of selection units for future reserves and actual vs targeted representation of features: western New South Wales its influence on a case study in New South Wales. Biological Conservation 85, 305-309.

Pressey, R.L., Bedward M., 1991. Mapping the environment at different scales: benefits and costs for nature conservation, in: C.R. Margules, Austin M.P. (Eds.), Nature Conservation: Cost Effective Biological Surveys and Data Analysis. CSIRO, Melbourne.

Pressey, R.L., Possingham, H.P., Logan, V.S., Day, J.R., Williams, P.H., 1999. Effects of data characteristics on the results of reserve selection algorithms. Journal of Biogeography 26, 179191.

Regan, H.M., Ensbey, M.J., Burgman, M., 2009. Conservation Prioritization and Uncertainty in Planning Inputs, in Moilanen, A., Wilson, K.A., Possingham, H.P. (Eds.), Spatial Conservation Prioritization. New York, Oxford University Press.

Rodrigues A.S.L., Gaston K.J., 2001. How large do reserve networks need to be? Ecology Letters 4, 602-09.

Rondinini, C., Wilson, K.A., Boitani, L., Grantham, H., Possingham, H.P., 2006. Tradeoffs of different types of species occurrence data for use in systematic conservation planning. Ecology Letters 9, 1136-1145.

Rouget, M., 2003. Measuring conservation value at fine and broad scales: implications for a diverse and fragmented region, the Agulhas Plain. Biological Conservation 112, 217-232.

Stockwell, D., Peterson, A.T., 2003. Comparison of resolution of methods used in mapping biodiversity patterns from point-occurrence data. Ecological Indicators 3, 213-221. 
Vazquez, L.B., Rodriguez, P., Arita, H.T., 2008. Conservation planning in a subdivided world. Biodiversity and Conservation 17, 1367-1377.

Warman, L.D., Sinclair, A.R.E., Scudder, G.G.E., Klinkenberg, B., Pressey, R.L., 2004. Sensitivity of systematic reserve selection about scale, biological data, and targets: case study from Southern British Columbia. Conservation Biology 18, 655-666.

Wiens, J.A., Bachelet, D., 2010. Matching the multiple scales of conservation with the multiple scales of climate change. Conservation Biology 25, 51-62.

Wiersma, Y.P., 2007. The effect of target extent on the location of optimal protected areas networks in Canada. Landscape Ecology 22, 1477-1487

Wilson, K.A., Westphal, M.I., Possingham, H.P., Elith, J., 2005. Sensitivity of conservation planning to different approaches to using predicted species distribution data. Biological Conservation $122,99-112$. 
Table 1. Average area and total number of planning units (n) for each grain size tested in this study. Also shown are the targets for each species distribution data type (occurrence or area of occupancy) used in the different conservation scenarios. Targets were set to make results from different grain sizes comparable.

\begin{tabular}{cccccc}
\hline \multicolumn{2}{c}{ Planning unit } & Data type: & Occurrence & Occurrence & Area $\left(\mathrm{km}^{2}\right)$ \\
\hline $\begin{array}{c}\text { Grain size } \\
\left(\mathrm{km}^{2}\right)\end{array}$ & $\mathrm{n}$ & Scenario: & $\begin{array}{c}\text { Constant } \\
\text { planning unit }\end{array}$ & $\begin{array}{c}\text { All other } \\
\text { scenarios }\end{array}$ & All scenarios \\
\hline 4.0 & 13992 & & 838 & 838 & 3363 \\
7.7 & 7412 & 838 & 436 & 3363 \\
14.7 & 3881 & 838 & 228 & 3363 \\
28.2 & 2032 & 838 & 119 & 3363 \\
66.1 & 865 & 838 & 51 & 3363 \\
130.9 & 437 & 838 & 26 & 3363 \\
197.9 & 289 & 838 & 17 & 3363 \\
273.6 & 209 & 838 & 12 & 3363 \\
430.0 & 133 & 838 & 8 & 3363 \\
602.0 & 95 & 838 & 6 & 3363 \\
1121.3 & 51 & 838 & 3 & 3363 \\
\hline
\end{tabular}


Table 2. Change in performance measures between the finest and coarsest grain sizes tested in each conservation scenario $(\%$ Change $=($ Value finest grain size- Value coarsest grain size $) /$ Value coarsest grain size)*100). Average values across all species are shown.

\begin{tabular}{lcccccc}
\hline & \multicolumn{2}{c}{ Efficiency } & \multicolumn{2}{c}{ Commission errors } & \multicolumn{2}{c}{$\begin{array}{c}\text { Conservation } \\
\text { Uncertainty }\end{array}$} \\
\cline { 2 - 7 } Scenario & Occurrence & Area & Occurrence & Area & Occurrence & Area \\
\hline Constant fine planning units & $-27.0 \%$ & $-26.2 \%$ & $81.1 \%$ & $81.7 \%$ & $91.3 \%$ & $90.9 \%$ \\
Constant coarse planning units & $3.3 \%$ & $1.2 \%$ & $81.9 \%$ & $80.9 \%$ & $90.9 \%$ & $90.8 \%$ \\
Constant fine distribution & $-27.5 \%$ & $-27.6 \%$ & $81.9 \%$ & $93.2 \%$ & $90.9 \%$ & $96.5 \%$ \\
Constant coarse distribution & $3.3 \%$ & $5.4 \%$ & $0.6 \%$ & $1.1 \%$ & $9.4 \%$ & $7.6 \%$ \\
Mixed & $-27.5 \%$ & $-27.0 \%$ & $81.9 \%$ & $81.0 \%$ & $90.9 \%$ & $91.3 \%$ \\
\hline
\end{tabular}


Figure 1. Conceptual representation of different combinations of grain sizes used for planning units and species distribution data (a) for a subcatchment (highlighted) of the Daly River basin within northern Australia (b). The shaded area represents the hypothetical distribution of a particular species. The finest grain size of species distribution data (left maps) was used to scale up predictions to coarser grain sizes (right maps). In this way, whenever a species was present in a finer scale planning unit contained within a coarser planning unit, that species was assumed to occupy the entire extent of the coarser planning unit.

Figure 2. Example calculations for two performance measures on an intermediate grain size. (a) Uncertainty in species distribution was assessed as 1 - the ratio of the area occupied at the fine scale / area expected at the coarse scale for those planning units where the species occurs (e.g. planning units $1-3)$. In this example the species occupies $0.22,0.40$ and 0.25 of planning units, so the uncertainty in its distribution for this grain size is 0.71 (i.e. 1-0.29). (b) Commission errors were assessed as the proportion of the expected species' representation not achieved. In this example, the species was expected to occupy all of planning unit 1 (the only planning unit included in the best solution where the species occurred), but it only occurred in a small proportion of the total area (0.22), so we can infer a commission error of 0.88 (i.e. 1-0.22).

Figure 3. Change in expected area of occupancy for three different species in the Daly River at three different grain sizes. The spatial distribution of each species at each grain size was obtained by scaling up predictions made at the finest scale and attributing this data to the different planning unit grain sizes.

Figure 4. Effect of grain size on performance measures for the five different conservation scenarios. See methods for more details on how each performance measure was assessed. Trends in average values across all species are shown.

Figure 5. Effect of grain size on performance measures for the three different species (shown in Figure 3) under the "mixed" scenario. See methods for more details on how each performance measure was quantified. 